\title{
Effect of Crop Growth and Canopy Filtration on the Dynamics of Plant Disease Epidemics Spread by Aerially Dispersed Spores
}

\author{
F. J. Ferrandino
}

Department of Plant Pathology and Ecology, The Connecticut Agricultural Experiment Station, P.O. Box 1106, New Haven 06504. Accepted for publication 15 January 2008.

\begin{abstract}
Ferrandino, F. J. 2008. Effect of crop growth and canopy filtration on the dynamics of plant disease epidemics spread by aerially dispersed spores. Phytopathology 98:492-503.

Most mathematical models of plant disease epidemics ignore the growth and phenology of the host crop. Unfortunately, reports of disease development are often not accompanied by a simultaneous and commensurate evaluation of crop development. However, the time scale for increases in the leaf area of field crops is comparable to the time scale of epidemics. This simultaneous development of host and pathogen has many ramifications on the resulting plant disease epidemic. First, there is a simple dilution effect resulting from the introduction of new healthy leaf area with time. Often, measurements of disease levels are made pro rata (per unit of host leaf area or total root length or mass). Thus, host growth will reduce the apparent infection rate. A second, related effect, has to do with the so-called "correction factor," which accounts for inocu-

tion and the initial exponential growth slows. Crop growth delays the impact of this limiting effect and, therefore, tends to increase the rate of disease progress. A third and often neglected effect arises when an increase in the density of susceptible host tissue results in a corresponding increase in the basic reproduction ratio, $R_{0}$, defined as the ratio of the total number of daughter lesions produced to the number of original mother lesions. This occurs when the transport efficiency of inoculum from infected to susceptible host is strongly dependent on the spatial density of plant tissue. Thus, crop growth may have a major impact on the development of plant disease epidemics occurring during the vegetative phase of crop growth. The effects that these crop growth-related factors have on plant disease epidemics spread by airborne spores are evaluated using mathematical models and their importance is discussed. In particular, plant disease epidemics initiated by the introduction of inoculum during this stage of development are shown to be relatively insensitive to the time at which inoculum is introduced.
\end{abstract} lum falling on already infected tissue. This factor accounts for multiple infection and is given by the fraction of the host tissue that is susceptible to disease. As an epidemic develops, less and less tissue is open to infec-
Additional keywords: mass-action, spore deposition.
During the vegetative growth phase of an annual field crop, ground cover or light interception increases logistically with time, and the leaf area, after an initial exponential growth period, increases approximately linearly with time $(12,18)$. The maximum growth rate is such that a square meter of leaf area per unit of ground area is produced every 5 to 7 days (19). This vegetative phase, depending on the crop, lasts from between 30 and 50 days, during which time leaf area density often increases by a factor of 4 to 6 . Toward the end of the growing season, leaves grow more slowly as carbohydrate is allocated to the storage and reproductive parts of the plant and senescent leaves are shed. One way to describe the overall behavior of a growing crop is to use the logistic equation asymptotically approaching a maximum leaf area (36). Waggoner (38) reported logistic crop growth rate parameters, $r_{c}\left(\mathrm{~d}^{-1}\right)$, varying from 0.06 to $0.24 \mathrm{~d}^{-1}$. For comparison, logistic fits to disease progress curves yield a range for Vanderplank's $r\left(\mathrm{~d}^{-1}\right)$ varying from 0.05 to $0.5 \mathrm{~d}^{-1}$ (37). Thus, the relative rate of growth and timing of disease development compared with crop growth will probably play an important role in the temporal development of plant diseases.

Almost all mathematical models describing the time course of an epidemic are based on some form of the following product law:

$$
\frac{d Y}{d t}=\alpha \cdot S \cdot H_{S}
$$

Corresponding author: F. J. Ferrandino

E-mail address: francis.ferrandino@po.state.ct.us

doi:10.1094/PHYTO-98-5-0492

(C) 2008 The American Phytopathological Society where $Y$ is some measure of the cumulative total infected tissue and $\alpha$ is a rate constant. The underlying assumption is that the number of new infections per unit of time is directly proportional to some measure of the amount of infective (sporulating) tissue, $S$, multiplied by some measure of the amount of healthy susceptible tissue, $H_{S}(1,11,37)$. The analogy has been drawn between equation 1 and the principle of mass-action used to describe the dynamics of chemical reactions $(10,23,39)$. The rate constant, $\alpha$, in equation 1 represents a number of different processes: propagule production, dispersal, deposition to new host sites, survival over the course of this process, and the eventual production of new infections. Host growth may affect any or all of these processes. Furthermore, it is well known that the ability of a pathogen to infect a host can be a strong function of the phenological age of the attacked tissue $(2,4,8,14-16,25)$. Due to this ontogenic resistance, a portion of the standing healthy plant area may not be susceptible to disease. Therefore, the number of susceptible and still healthy standing sites, $H_{S}$, will explicitly depend on the age distribution of the host sites (11), which is determined by the time course of crop growth.

If $S$ and $H_{S}$ in equation 1 are expressed in terms of number of infection sites, then the product $S \cdot H_{S}$ represents the total number of sporulating site-healthy site pairs in the population, which represent every possible pathway by which a propagule of disease can result in a new infection. For a field of fixed area, such a formulation is consistent with observations showing that apparent infection rates are dependent on initial plant density $(5,7)$. The above assumption is equivalent to what Anderson et al. (1) and de Jong et al. (10) call "pseudo mass-action." There are many reasons why this simple assumption may lead us astray. For plant diseases disseminated by airborne spores, the deposition of pro- 
pagules to plant foliage is definitely not a simple linear function of susceptible sites as implied above $(3,12)$. On the other hand, if $H_{S}$ in equation 1 is expressed as the fraction of the total number of infection sites that are healthy and susceptible, then the resulting epidemic model is based on what Anderson et al. (1) and de Jong et al. (10) call "true mass-action" $(1,10)$. In this case, any effect of plant growth on disease progress is implicit in the factor $\alpha$. There has been some discussion in the literature $(17,28)$ as to appropriate use of the above nomenclature. In the ecological literature (28), the pseudo mass-action case is characterized by densitydependent transmission, whereas the true mass-action case is dependent on frequency-dependent transmission. Irrespective of the names given to these models, they are based on, perhaps, unacceptably simple mathematical abstractions from reality. McCallum et al. (28) suggest that a more realistic epidemic model should reflect the physical mechanisms of the transmission of disease.

The fate of airborne spores is the result of a three-way competition between deposition to the ground, deposition to plants, and escape from the field. The relative rates of these competing processes are governed by leaf area density, the gravitational settling speed of the spores, as well as wind speed and turbulence within and above the plant canopy. In fact, as I will show, this competitive system follows Michaelis-Menten kinetics (29) with respect to leaf area density, so that the probability of infection per host site decreases as the number of host sites increases. This is due to the fact that leaves, in a dense canopy, shield one another from the flux of airborne spores. Furthermore, if spore survival is dependent on canopy density, the spore survival fraction is also explicitly dependent on the number of host sites. The net result is that the parameter $\alpha$ in equation 1 has a strong functional dependence on crop growth and is definitely not constant in time. At low foliar densities, the amount of spores deposited increases with increasing leaf area and pseudo mass-action is the appropriate model. Later in the season, for dense plant canopies, spore catch on leaves approaches a maximum value and true mass-action describes the situation.

The purpose of this paper is to demonstrate that pseudo massaction and true mass-action epidemic models are really extreme cases of a range of more realistic epidemic models based on crop density-dependent inoculum transmission. To achieve this goal, I incorporate a crop growth model $(12,18)$ and an estimate for canopy filtration efficiency into the derivation of an epidemic model. Leaf area as a function of time is assumed to follow a logistic law. The relationship of leaf area to canopy filtration efficiency is estimated using dimensional analysis and a gradient diffusion approximation (K-theory) for turbulent transport (3). The results of model calculations are then used to evaluate the ramifications of crop growth on the basic reproduction ratio, $R_{0}$ (defined as the ratio of the total number of daughter lesions produced to the number of original mother lesions), of aerially dispersed pathogens and on the temporal development of the ensuing epidemic. The variables and parameters used are listed in Table 1.

\section{THEORY AND APPROACHES}

Epidemic models: previous work and present outlook. Mass-action models. The product law (equation 1) has three population variables: $Y, S$, and $H_{S}$, as well as a rate parameter $\alpha$, which is usually assumed to be a constant. Thus, a closed form solution to equation 1 will depend on our ability to express two of these variables in terms of the remaining one. The specification of $S$ involves some assumption about the phenology of infected tissue and will, in general, depend on the time course of infection. By analogy, the specification of $H_{S}$ involves some assumption about the phenology and susceptibility of plant tissue to disease and will depend on the time course of crop growth.

Vanderplank (37) solved this problem by ignoring crop growth and assuming that a newly infected host site is latent for a discrete time period $p$ and then remains infectious for a time $i$. Since the total number of host sites, $N_{0}$, was assumed to be constant, equation 1 could be divided through by this value and expressed in terms of fractional values. Alternatively, compartment models characterize the total number of host sites, $N$, as either being healthy, $H$, latently infected, $L$, sporulating, $S$, or removed, $R$, such that $N=H+L+S+R$ and $Y=L+S+R(9,21,26,32)$. The development of the epidemic is then expressed as a series of coupled linear differential equations (35). Recently, it has been shown that both the Vanderplank discrete time model and the compartmental models fit into a larger theoretical framework of the classic Kermack-McKendrick epidemic model $(23,27,35)$. Following Diekman et al. (11) and Heesterbeek (20), we now define the basic reproduction ratio, $R_{0}$, as "the expected number of secondary cases produced, in a completely susceptible population, by a typical infected individual during its entire period of infectiousness." In addition, we assume that the rate of spore production per lesion can be expressed as a function $I(\tau)$ (spores $\mathrm{d}^{-1}$ lesion $^{-1}$ ) of lesion age, $\tau(\mathrm{d})$. With the above assumptions and definitions, the Kermack-McKendrick epidemic model results in a disease progress curve that is totally determined by $R_{0}$, the shape of the sporulation curve $(I(\tau))$, and the initial conditions $(19,35)$. However, what happens to the above analysis when the crop grows and the rate parameter $\alpha$ is a function of time?

Explicit models. We can, of course, examine the problem numerically and include the time dependence of host tissue susceptibility to disease as well as crop growth and variable $\alpha$. There are many numerical plant disease models in the literature based on either the true mass-action or the pseudo mass-action assumption $(6,22,24,25,34,40)$. These models account for the effect of crop growth as an increase in the amount of susceptible healthy tissue, but do not include the increased efficiency by which airborne spores are captured by plant tissue as foliar density increases. Ferrandino (12) presented a combined analytical/numerical model for the spread of late blight on potato and its effects on yield, which included the rudiments of canopy filtration.

In what follows, I seek analytical solutions for disease development, which include the effects of crop density on disease transmission to illuminate both the physical and biological nature of the infection process. To this end, following Vanderplank (37), I will make the following simplifying assumptions.

- $S$, the number of sporulating sites, is a constant fraction, $f_{R}$, of $Y$, the total number of infected sites.

- All healthy sites, $H$, are susceptible to disease.

- Ignoring removals, the total number of sites, $N$, is equal to the sum of $H$ and $Y$.

In this description, sites could be infection courts, leaflets, leaves, stems, or whole plants. The above assumptions lead to three relations among $Y, S, H, H_{S}$, and $N$ :

$$
\begin{aligned}
& S=f_{R} \cdot Y \\
& H=N-Y\} \\
& H_{S}=H
\end{aligned}
$$

which when combined with equation 1 yield

$$
\frac{d Y}{d t}=\alpha f_{R} Y(N-Y)
$$

The general solution to equations of the above form (Appendix equation A1) can be obtained by quadrature or direct integration if the quantities $\alpha f_{R}$ and $N$ are given as functions of time (Appendix equations A3 to A5). For the special case where $N=N_{0}$ is a constant, equation 3 can be divided by $N_{0}$ to yield

$$
\frac{d y}{d t}=r y(1-y)
$$

where the diseased fraction, $y$, is defined by $y=Y / N_{0}$ and Vanderplank's rate constant, $r\left(\mathrm{~d}^{-1}\right)$, is set equal to the product, 
$\alpha f_{R} N_{0}$. If $r$ is assumed constant, then the solution to equation 4 results in a logistic epidemic (Appendix equation A7).

In what follows, I will assume that the total number of host sites, $N$, is similarly given by a logistic equation which asymptotically approaches a maximum value of $N_{\max }$ with the logistic rate of crop growth denoted by $r_{c}\left(\mathrm{~d}^{-1}\right)$, such that

$$
\frac{d N}{d t}=r_{c} N \frac{N_{\max }-N}{N_{\max }}
$$

True mass-action. In order to account for logistic crop growth, by simultaneously solving equations 3 and 5, Waggoner (38) assumed that the quantity $r_{W}\left(\mathrm{~d}^{-1}\right)=\alpha f_{R} N$ remains constant for a

TABLE 1. Symbols

\begin{tabular}{|c|c|c|}
\hline Symbol & Units $^{\mathrm{a}}$ & Definition \\
\hline$a$ & $\mathrm{~m}^{2}$ & The area of a site \\
\hline$A$ & ND & Leaf area index (leaf area per unit ground area) \\
\hline$A_{H}$ & ND & Healthy host leaf area per unit ground area \\
\hline$A_{\max }$ & ND & Maximum leaf area per unit ground area in logistic crop growth model \\
\hline$A_{Y}$ & ND & Infected host leaf area per unit ground area \\
\hline$b_{1}$ & {$[X]^{-1} s^{-1}$} & Rate parameter in equation $\mathrm{A} 1$ \\
\hline$b_{2}$ & {$[X]$} & Limit parameter in equation $\mathrm{A} 1$, corresponding to the maximum value of the arbitrary function $X$ \\
\hline$C$ & $N_{\text {sp }} \mathrm{m}^{-3}$ & Aerial spore concentration \\
\hline$C F$ & & Acronym for canopy filtration \\
\hline COFR & ND & The correction factor $(H / N$, literature citation 40$)$ \\
\hline$C_{1}, C_{2}, C_{3}$ & ND & Parameters defined to simplify the equations A16 to A26 \\
\hline$f_{h}$ & ND & Horizontally projected fraction of leaf area \\
\hline$f_{p}$ & ND & Canopy filtration efficiency, defined as the probability that an airborne spore is deposited on foliage \\
\hline$f_{R}$ & ND & Fraction of infected sites which are reproductive \\
\hline$F_{A}$ & $\mathrm{~m}^{2}$ & The area of the field under study \\
\hline$h$ & $\mathrm{~m}$ & Crop height \\
\hline$H$ & $N_{s t}$ & Total number of healthy sites \\
\hline$H_{0}$ & $N_{s t}$ & Initial number of healthy sites \\
\hline$H_{S}$ & $N_{s t}$ & Total number of susceptible healthy sites \\
\hline$I(\tau)$ & $N_{s p} N_{s t}{ }^{-1} \mathrm{~d}^{-2}$ & The time rate of spore production per lesion as a function of lesion age \\
\hline$I F(t)$ & ND & Integrating factor which renders equation A2 exact (Appendix) \\
\hline$J_{E}$ & $N_{s p} \mathrm{~m}^{-1} \mathrm{~s}^{-1}$ & Flux of spores escaping from the plant canopy \\
\hline$J_{G}$ & $N_{s p} \mathrm{~m}^{-1} \mathrm{~s}^{-1}$ & Flux of spores deposited to the ground \\
\hline$J_{P}$ & $N_{s p} \mathrm{~m}^{-1} \mathrm{~s}^{-1}$ & Flux of spores deposited to the plant canopy \\
\hline$k$ & ND & von Karman's constant $(30, k=0.4)$ \\
\hline$K_{M}$ & $\mathrm{~m}^{2} \mathrm{~s}^{-1}$ & Eddy diffusivity for momentum (30) \\
\hline$L$ & $N_{l e}$ & Number of latently infected sites \\
\hline$N$ & $N_{s t}$ and $N_{l e}$ & Total cumulative number of standing sites, including healthy and infected sites \\
\hline$N_{0}$ & $N_{s t}$ and $N_{l e}$ & Assumed constant number of standing sites, including healthy and infected sites \\
\hline$N_{\max }$ & $N_{s t}$ and $N_{l e}$ & Maximum number of standing sites, including healthy and infected sites \\
\hline$n$ & ND & Normalized leaf area per unit ground area $\left(n=A / A_{\max }\right)$ \\
\hline PMA & & Acronym for pseudo mass-action \\
\hline$r$ & $\mathrm{~d}^{-1}$ & Vanderplank's rate constant \\
\hline$r_{c}$ & $\mathrm{~d}^{-1}$ & Logistic rate parameter for crop growth model \\
\hline$r_{C F}$ & $\mathrm{~d}^{-1}$ & Rate constant for canopy filtration model \\
\hline$r_{P}$ & $d^{-1}$ & Rate constant for pseudo mass-action model \\
\hline$r_{W}$ & $\mathrm{~d}^{-1}$ & Rate constant for Waggoner's true mass-action model \\
\hline$R$ & $N_{s t}$ & Total cumulative number of removed sites \\
\hline$R_{0}$ & ND & $\begin{array}{l}\text { The basic reproduction ratio, defined as the ratio of the total number of daughter lesions produced to the number of original } \\
\text { mother lesions }\end{array}$ \\
\hline $\mathrm{S}$ & $N_{l e}$ & Number of infective actively sporulating sites \\
\hline$t_{\text {start }}$ & $\mathrm{d}$ & Time at which a disease lesion is initiated \\
\hline$T_{E}$ & $\mathrm{~s}$ & Characteristic time for escape from the plant canopy \\
\hline$T_{G}$ & $\mathrm{~s}$ & Characteristic time for deposition to the ground \\
\hline TMA & & Acronym for true mass-action \\
\hline$T_{P}$ & $\mathrm{~s}$ & Characteristic time for deposition to plant material \\
\hline$u(z)$ & $\mathrm{m} \mathrm{s}^{-1}$ & Horizontal wind speed \\
\hline$u_{*}$ & $\mathrm{~m} \mathrm{~s}^{-1}$ & The friction velocity (30) \\
\hline$v_{g}$ & $\mathrm{~m} \mathrm{~s}^{-1}$ & Gravitational settling speed of airborne spores \\
\hline$X$ & {$[X]$} & Arbitrary function of time in equation A1 \\
\hline$Y$ & ND & The diseased fraction $(y=Y / N)$ \\
\hline$Y$ & $N_{l e}$ & Total cumulative number of infected sites, including latent infections, infectious sites, and removals: $Y=L+S+R$ \\
\hline$z$ & $\mathrm{~m}$ & Height \\
\hline$z_{0}$ & $\mathrm{~m}$ & Roughness scale (33) \\
\hline$\alpha$ & $N_{s t}^{-1} \mathrm{~s}^{-1}$ & Rate constant for the law of mass action (equation 1) \\
\hline$\beta$ & ND & The assumed constant value for the canopy filtration efficiency, $f_{P}$, in the true mass-action model \\
\hline$\gamma$ & ND & The assumed constant of proportionality in the equation: $f_{P}=\gamma N / N_{\max }$ in the pseudo mass-action model \\
\hline$\delta$ & ND & $\begin{array}{l}\text { The ratio of the number of spores lost to both the ground and the air above the canopy to the number of spores deposited } \\
\text { on plant tissue }\end{array}$ \\
\hline$\xi$ & $N_{s t}^{-1}$ & $\begin{array}{l}\text { Probability per healthy host site that a spore is liberated, becomes airborne, and is physically transported and deposited to } \\
\text { such a site }\end{array}$ \\
\hline$\tau$ & $\mathrm{d}$ & Age of lesion \\
\hline$\tau_{\text {start }}$ & $\mathrm{d}$ & Time at which inoculum is introduced into a field \\
\hline$\psi$ & $N_{l e} N_{s p}^{-1}$ & Fraction of spores that having landed on susceptible host cause a new lesion \\
\hline
\end{tabular}

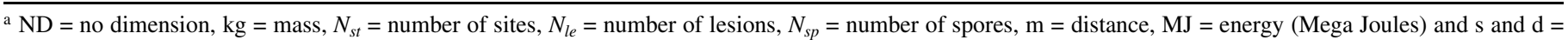
time. 
logistically growing crop. The above assumption is equivalent to what Anderson et al. (1) and de Jong et al. (10) call true massaction. Hereafter, this model is denoted by TMA. In essence, the parameter $\alpha$ in equations 1 and 3 is assumed to be inversely proportional to the size of the host population, $N$. With these assumptions, equation 3 becomes

$$
\frac{d Y}{d t}=\alpha f_{R} Y(N-Y)=r_{W} Y \frac{(N-Y)}{N}
$$

The development of disease in such a situation is modeled using two coupled nonlinear differential equations (equations 5 and 6) which can be solved analytically (Appendix equations A8 and A9; Ferrandino's Appendix in literature citation 38).

Pseudo mass-action. Alternatively, one can assume that the $\alpha$ in equations 1 and 3 is a constant. The above assumption is equivalent to what Anderson et al. (1) and de Jong et al. (10) call pseudo mass-action. Hereafter, this model is denoted by PMA. Letting $r_{P}$ $\left(\mathrm{d}^{-1}\right)=\alpha f_{R} N_{\text {max }}$, equation 3 becomes

$$
\frac{d Y}{d t}=\alpha f_{R} Y(N-Y)=r_{P} Y \frac{(N-Y)}{N_{\max }}
$$

The development of disease in such a situation is once again modeled using two coupled nonlinear differential equations (equations 5 and 7). The time integrals in the quadrature solution to equation 5 coupled to equation 7 can be written in closed form when $r_{P}$ is an integer multiple of $r_{c}$ (Appendix equations A10 to A12).

Note that if the total number of sites, $N$, is constant then both equation 6 and equation 7 revert to equation 3 . As will be shown below for aerially dispersed pathogens, Waggoner's true massaction approach (equation 6) is appropriate for dense canopies or low wind speeds when spore deposition is transport-limited (13). In this case, most of the airborne spores are deposited to foliage, independent of leaf area. The pseudo mass-action compatible approach (equation 7) is appropriate for sparse canopies or high wind speeds when spore deposition is sink-limited (13). In this case, most of the spores are either deposited to the ground or escape from the plant canopy, and spore deposition to foliage increases linearly with leaf area. In reality, spore deposition in a growing crop will lie somewhere between the above two extremes depending on wind, within canopy turbulence, and changing foliar density.

Repercussions of the finite nature of spore production. Consider the pseudo mass-action form of equation 1 with all population variables $\left(Y, S, H=H_{S}\right)$ expressed as sites or sites per unit of ground area. As long as vegetation is sparse we would expect the per diem rate of new infections to increase with the product of the number of sporulating sites, $S$, and the number of uninfected susceptible sites, $H$. However, as foliar density increases a difficulty with this simple product law arises. To illustrate the problem, assume that only one site in an entire field of plants is infected and sporulating. This sporulating site ( $\mathrm{S}$ site) liberates a

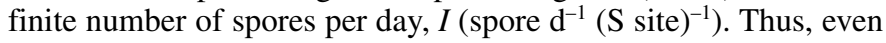
under the most optimal conditions, such that every spore produced results in a new infected site, a maximum of $I$ new infected sites can be produced per diem. However, according to equation 1 , if we continue to increase the size of the field, the number of target sites, $H$ (T site), will increase and the number of lesions produced per day, $\alpha H$ (remember $S=1$ ), will continue to increase and eventually become larger than $I$. Obviously, the plants cannot catch more spores than are released, so that the law of pseudo mass-action breaks down at high plant densities, and the parameter $\alpha$ cannot remain constant independent of the number of healthy sites, $H$. Models based on the true mass-action assumption avoid this difficulty through the assumption that the parameter $\alpha$ is inversely proportional to $N$, the total number of sites. Under this unrealistic assumption, disease progress is not expli- citly dependent on crop growth and the plant canopy intercepts a constant fraction of the airborne inoculum independent of leaf area density.

As stated above, the rate constant, $\alpha$, in equation 1 represents a number of different processes. For aerially dispersed spores, $\alpha$ can be expressed as the product of three quantities: the time rate of aerial propagule production per infectious site (I [spore (S site $\left.)^{-1} \mathrm{~d}^{-1}\right]$ ), the probability of an airborne spore being deposited on a healthy site per healthy site $\left(\xi\left[(\mathrm{T} \text { site })^{-1}\right]\right)$, and the fraction of the spores deposited on healthy sites that go on to produce a new infected site $\left(\psi\right.$ [lesion spore $\left.\left.{ }^{-1}\right]\right)$. To analyze the implications of nonconstant $\alpha$, we will first reexamine the dimensionality of equation 1 and the role of the parameter $\alpha$ :

$$
\begin{aligned}
& \frac{d Y}{d t}=\quad \alpha \quad \cdot S \cdot H \\
& {\left[\frac{\text { lesion }}{\text { day }}\right]=\left[\frac{\text { lesion }}{\text { day } \cdot S \text { site } \cdot T \text { site }}\right] \cdot[S \text { site }] \cdot[T \text { site }]}
\end{aligned}
$$

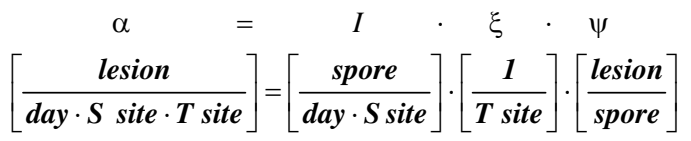

The nomenclature of equation 8 is consistent with that of Segarra et al. (35). The dimensionality of the parameter $\xi$ is problematical. For an epidemic in a growing crop the number of healthy sites is simultaneously decreased by new infections and increased by crop growth. Since $\xi$ is defined per healthy site, this parameter is implicitly dependent on both the stage of the epidemic and the stage of crop growth. We need to make these dependencies explicit. If spores are deposited equally to every site irrespective of whether the site is infected or not, then the probability of an airborne spore being deposited on any site, $f_{P}$ (not dimensioned ND), divided by the total number of sites, $N$, must equal $\xi$ (i.e., $\xi=$ $\left.f_{P} / N\right)$. The product $\xi H$ in equation 1 , the fraction of spores which are deposited on healthy sites, can then be expressed as the product of $f_{P}$ and the fraction of all sites that are healthy, $H / N$. The canopy filtration efficiency, $f_{P}$, is dependent on the physical properties of the canopy and the mechanical properties of wind and turbulence and independent of epidemic development. The ratio, $H / N$, the fraction healthy sites, is what Zadoks (40) called the correction factor (COFR), which accounts for the effect of disease progress on the fractional spore deposition to healthy sites. A comparison of the above models (equations 6 and 7) with equation 8 leads us to the following conclusions.

- True mass-action. Equation 6 is based on the assumption that $f_{p}$ is a constant, so that $\alpha \propto I \psi / N$ and $r_{W} \propto I \psi f_{R}$. For later reference, this constant value for the canopy filtration efficiency will be denoted by $\beta$.

- Pseudo mass-action. Equation 7 is based on the assumption that the quantity, $f_{p}$, is proportional to the total number of sites, $N$. Letting $f_{p} \propto N$ gives $\alpha \propto I \psi$ and $r_{P} \propto I \psi f_{R}$. For later reference, the proportionality constant for the canopy filtration efficiency will be denoted by $\gamma$ such that $f_{p}=\gamma N / N_{\max }$.

Thus, the major difference between the above two models (equations 6 and 7) involves the assumed relationship between efficiency with which foliage captures airborne spores and the number of host sites.

Canopy filtration model. The information provided by $N(t)$, the number of host sites as a function of time, is inadequate to provide a mechanistic view of crop growth and spore dissemination. Other information about the host sites, the crop, and the field must be supplied, e.g., What is the site density per unit volume? How large are these host sites? How are they physically oriented? What is their spatial distribution? How large is the field? How tall is the crop?

Assuming that the area of a site is given by $a\left(\mathrm{~m}^{2}\right)$ and that there are a total of $N(t)$ sites at time, $t$, within a field of area $F_{A}$ 
$\left(\mathrm{m}^{2}\right)$, then the total leaf area per unit of ground area, $A$ (the leaf area index), the infected leaf area per unit of ground area, $A_{Y}$, and the healthy leaf area per unit of ground area, $A_{H}$, are given by

$$
A(t)=\frac{N(t) \cdot a}{F_{A}} ; \quad A_{Y}(t)=\frac{Y(t) \cdot a}{F_{A}} ; \quad A_{H}(t)=\frac{H(t) \cdot a}{F_{A}}
$$

The principle of mass-action, upon which equations 1 and 2 are based, is not appropriate for aerially dispersed pathogens within a rapidly growing field crop $(3,10,12)$. Mechanically, the fraction of airborne spores deposited on leaves is dependent on the leaf area index, which is a function of time. For that reason, in what follows all variables enumerating the number of host sites $(N, Y, H$, etc.) are multiplied by the ratio $a / F_{A}$ to express them as leaf area indices (equation 9).

The fate of airborne spores released from infective tissue within a crop depends on the relative rates of escape from the field, deposition to the ground, and deposition to plant material. Following Aylor (3), I draw the analogy to an electric circuit, where $J_{P}, J_{G}$, and $J_{E}$ are spore fluxes assumed directly proportional to the inverse of the time scales $T_{P}, T_{G}$, and $T_{E}$ (resistances) and corresponding to deposition on the plant canopy, deposition on the ground, and escape from the plant canopy, respectively. These three fluxes are in parallel, so that the fraction of spores landing on plant material, $f_{P}$ (the canopy filtration efficiency), is equal to the ratio of the spore flux deposited on the plants, $J_{P}$, divided by the total spore flux, $J_{P}+J_{G}+J_{E}$, such that

$$
f_{P}=\frac{J_{P}}{J_{P}+J_{G}+J_{E}}=\frac{\frac{1}{T_{P}}}{\frac{1}{T_{P}}+\frac{1}{T_{G}}+\frac{1}{T_{E}}}
$$

The evaluation of the canopy filtration efficiency, $f_{P}$, above depends on the deposition of airborne spores to foliar elements in the turbulent air within the plant canopy. If we assume that the airborne spores are distributed evenly throughout the plant canopy with aerial concentration, $C$ (spores $\mathrm{m}^{-3}$ ), and that deposition onto foliage is dominated by gravitational settling, then the spore flux onto plant surfaces and the ground are given by

$$
\left.\begin{array}{l}
J_{P}=v_{g} \cdot f_{h} \cdot A \cdot C \\
J_{G}=v_{g} \cdot C
\end{array}\right\}
$$

where $v_{g}\left(\mathrm{~m} \mathrm{~s}^{-1}\right)$ is the gravitational settling speed of spores and the product $f_{h} A$ is the horizontally projected leaf area per unit of ground area.

The time scale for escape from the plant canopy will depend on the wind and air turbulence within and above the crop. Above the canopy wind speed and turbulence are governed by MoninObukhov similarity theory (30). Above the plant canopy under neutrally stable conditions, wind speed, $u\left(\mathrm{~m} \mathrm{~s}^{-1}\right)$, varies logarithmically and eddy diffusivity for momentum, $K_{M}\left(\mathrm{~m}^{2} \mathrm{~s}^{-1}\right)$, varies linearly with aerodynamic height, $(z-d)(\mathrm{m})$ :

$$
u(z)=\frac{u_{*}}{k} \ln \left(\frac{z-d}{z_{0}}\right)
$$

and

$$
K_{M}(z)=k u_{*}(z-d)
$$

where $k(\mathrm{ND})$ is von Karman's constant $(k=0.4), u_{*}\left(\mathrm{~m} \mathrm{~s}^{-1}\right)$ is the friction velocity, $d(\mathrm{~m})$ is the zero plane displacement height, and $z_{0}(\mathrm{~m})$ is the roughness scale (33). In what follows, I assume that the eddy diffusivity for momentum, $K_{M}$, also applies to airborne spores (33). Thus, the time scale for escape from the plant canopy can be approximated as

$$
T_{E}=\frac{h^{2}}{\left.K_{M}\right|_{z=h}}=\frac{h^{2}}{k u_{*}(h-d)}=\frac{h}{0.12 \cdot u_{*}}
$$

where $h$ is the height of the plant canopy and the zero-plane displacement height, $d$, is assumed equal to $0.3 \cdot h$ (33). Simple dimensional analysis yields

$$
J_{E}=\frac{C \cdot h}{T_{E}}=0.12 \cdot u_{*} \cdot C,
$$

and the above approximations (equations 11 and 13) combined with equation 10 yield

$$
f_{P}=\frac{f_{h} A}{1+f_{h} A+\frac{0.12 \cdot u_{*}}{v_{g}}}=\frac{n}{n+\delta}=\left(1+\frac{\delta}{n}\right)^{-1}
$$

where

$$
\delta=\frac{v_{g}+0.12 \cdot u_{*}}{v_{g} f_{h} A_{\max }}
$$

and $n=A / A_{\max }=N / N_{\max }$. Equation 15 has the same form as the Michaelis-Menten equation (29), wherein, the rate of reaction for a substrate of concentration $A$ is proportional to $A$ divided by the sum of a constant and $A$. The parameter $\delta$ is the ratio of the number of spores lost to both the ground and the air above the canopy to the number of spores deposited on plant tissue. The value of $\delta$ is of the order of unity, which can be seen by inserting reasonable values into the above definition (for $v_{g}=0.01 \mathrm{~m} \mathrm{~s}^{-1}$, $u_{*}=0.2 \mathrm{~m} \mathrm{~s}^{-1}, f_{h}=0.5, A_{\max }=6$, and $\delta=1.13$ ). When the leaf area is very small, pseudo mass-action applies, i.e., $f_{P}$ is approximately proportional to leaf area. However, as the crop grows, leaf area increases, and $f_{P}$ approaches the maximum value of $(1+\delta)^{-1}$. So that, for dense canopies, $f_{P}$ is a constant and true mass-action applies. Hereafter, this canopy filtration model is denoted by $\mathrm{CF}$. Combining equations $3,8,9$, and 15 yields

$$
\frac{d A_{Y}}{d t}=\frac{I \psi f_{R} n}{(n+\delta)} A_{Y} \frac{\left(A-A_{Y}\right)}{A}=\frac{r_{C F} n(1+\delta)}{(n+\delta)} A_{Y} \frac{\left(A-A_{Y}\right)}{A}
$$

where $r_{C F}\left(\mathrm{~d}^{-1}\right)=I \psi f_{R} /(1+\delta)$. Alternatively, using equation 9 , the above equation (equation 16) can be expressed in terms of sites as

$$
\frac{d Y}{d t}=\frac{I \psi f_{R} n}{(n+\delta)} Y \frac{(N-Y)}{N}=r_{C F} \frac{(1+\delta) n}{(n+\delta)} Y \frac{(N-Y)}{N}
$$

The time integrals in the quadrature solution to equation 5 coupled to equation 17 can be written in closed form when $r_{C F}$ is an integral multiple of $r_{c}$ (Appendix equations A17 and A18).

\section{RESULTS}

Model calculations. A comparison of models. The above three models (TMA [equation 6], PMA [equation 7], and CF [equation 17]) differ only in the assumed expression for $f_{P}$ (Table 2). The two arbitrary parameters, $\beta$ and $\gamma$, for the PMA and TMA models, respectively, can be chosen such that all three models give the same results for late season epidemics (Table 2: $\beta=\gamma=(1+\delta)$ ). The role of the parameter $\delta$ (equation 15) in determining the canopy filtration efficiency for the $\mathrm{CF}$ model is illustrated in Figure 1 . Note $f_{P}$ for the CF model is bounded by the assumptions of the other two models (PMA and TMA). The solutions of the three models (TMA [equation 6], PMA [equation 7], and CF [equation 17] with $r_{W}=r_{P}=r_{C F}=2 r_{c}, Y_{0} / N_{\max }=0.00167, n_{0}=$ $0.01, \delta=1$ ) are plotted in Figure 2. Due to the low spore catch when leaf area is small for both the PMA and CF models, plant disease epidemics are delayed until the canopy starts to grow. These mass-action-based epidemics are characterized by accelerating logistic growth rates and are negatively skewed in time, as illustrated in Figure 2. Note that all three models degenerate to the same simple logistic equation for epidemics starting at a time, $\tau_{\text {start }}=10 / r_{c}$, after the vegetative period for which $n$ is very close to unity. 
The diseased fraction $y$. Experimentally, one does not directly measure the number of infected sites, $Y$. Usually, a sample (a collection of leaves, stems, or plants) is assayed for disease to obtain the diseased fraction, $y=Y / N$. Diseased fractions for the epidemics shown in Figure 2 are plotted in Figure 3. The initial decrease in the diseased fraction for the PMA and CF models is due to the dilution effect of the growing crop. This effect is also what causes the time lag in these epidemics. Basically, the crop must grow to a certain size before enough spores are captured so that disease increases at least as fast as the crop grows. To put the above statement into more formal terms we must reexamine the model equations.

Our next task is to describe the time course of disease development in terms of $y$ when the crop is growing. Combining equations 5 and 6 with the definition of the derivative of the quotient, $Y / N$, yields

$$
\frac{d y}{d t}=\frac{d(Y / N)}{d t}=\frac{1}{N} \frac{d Y}{d t}-\frac{Y}{N^{2}} \frac{d N}{d t}=r_{W} y(1-y)-r_{c} y(1-n)
$$

where $n=N / N_{\max }$ and the negative term on the right side of equation 18 accounts for dilution due to crop growth. Setting the derivative in equation 18 equal to zero, we define the critical disease severity, $y_{\text {crit }}$, as a function of time:

$$
y_{\text {crit }}=1-\frac{r_{c}(1-n)}{r_{W}}=1-\frac{r_{c}(1-n(0))}{r_{W} n(0)} \frac{\exp \left(-r_{c} t\right)}{\left[1+\frac{(1-n(0))}{n(0)} \exp \left(-r_{c} t\right)\right]}
$$

where the logistic solution for crop growth (Appendix equation A7) has been substituted for $n(t)$. Analogous analyses for the pseudo mass-action case (PMA: equations 5 and 7) yield

$$
\frac{d y}{d t}=\frac{d(Y / N)}{d t}=\frac{1}{N} \frac{d Y}{d t}-\frac{Y}{N^{2}} \frac{d N}{d t}=r_{p} n y(1-y)-r_{c} y(1-n)
$$

and

$$
y_{\text {crit }}=1-\frac{r_{c}}{r_{p}} \frac{(1-n)}{n}=1-\frac{r_{c}(1-n(0))}{r_{p} n(0)} \exp \left(-r_{c} t\right)
$$

and for the canopy filtration case (CF: equations 5 and 17), the results are

$$
\frac{d y}{d t}=r_{C F} \frac{(1+\delta) n}{(n+\delta)} y(1-y)-r_{c} y(1-n)
$$

where $y=Y / N=A_{Y} / A, n=N / N_{\max }=A / A_{\text {max }}$, and $y_{\text {crit }}$ is given by

$y_{c r i t}=1-\frac{r_{c}}{r_{C F}} \frac{(1-n)(n+\delta)}{(1+\delta) n}=1-\frac{r_{c}}{r_{C F}} \frac{[1-n(0)]}{n(0)} \cdot \frac{\exp \left(-r_{c} t\right)\left[1+\frac{\delta[1-n(0)]}{(1+\delta) n(0)} \exp \left(-r_{c} t\right)\right]}{\left[1+\frac{[1-n(0)]}{n(0)} \exp \left(-r_{c} t\right)\right]}$

The solution to equation 22 is illustrated in Figure 4A for the CF model where $y$ is plotted versus nondimensional time $r_{c} t$. The short line segments in Figure 4A represent the derivative in equation 22 and the hatched line is a plot of $y_{\text {crit }}$ (equation 23). For disease severities less than the critical value (equation 23), the diseased fraction increases with time. For disease severities greater than the critical value (equation 23), the crop grows faster than the epidemic and the fractional disease severity decreases with time. At the critical value, both disease levels and crop density grow at the same rate, so that the diseased fraction remains constant. The relationships among the values of $y_{\text {crit }}$ for all of the models (TMA, equation 19; PMA, equation 21, and CF, equation 23) are illustrated in Figure 4B. Note that the canopy filtration model smoothly transforms from the PMA case $(\delta \rightarrow 0)$ to the MA case $(\delta \rightarrow \infty)$ as the parameter $\delta$ increases.

The time lag. The initial behavior of the three models is examined in the Appendix. The TMA model corresponds to an increase of $Y$ over time (equation A22). However, the behavior of the other two models is more complex. Due to the low efficiency of foliar spore capture in the early stages of crop growth, disease progress is stifled for the PMA and CF models when compared with the TMA model. The net result (Appendix equations A19 to A26) is that the initial epidemic curves for the PMA and CF models approach exponential curves after periods of time, $\tau_{P}$ and $\tau_{C F}$, respectively, given by

$$
\tau_{P}=-\frac{\ln [n(0)]}{r_{c}}
$$

and

$$
\tau_{C F}=-\frac{\ln \left[\frac{n(0)(1+\delta)}{n(0)+\delta}\right]}{r_{c}}=-\frac{\ln \left(C_{1}\right)}{r_{c}}
$$

where $C_{1}$ is defined in equation A16. This behavior is independent of the initial level of disease $[Y(0)$ or $y(0)]$. For comparison, consider the time it takes for a logistically growing crop to attain half its maximum leaf area, $\tau_{c^{1 / 2}}$, given by

$$
\tau_{c 1 / 2}=-\frac{\ln \left[\frac{n(0)}{1-n(0)}\right]}{r_{c}}
$$

If the initial value of $n$ is small $(n<0.1)$, the two time scales $\tau_{P}$ (equation 24) and $\tau_{c^{1 / 2}}$ (equation 26) are almost identical, with $\tau_{P} \sim$ $\tau_{c^{1 / 2}}+n(0) / r_{c}$. The time lags for CF models (equation 25) are smaller by a factor depending on the value of the parameter $\delta$. For example, if $n(0)=0.01$ and $\delta=1$, then $r_{c} \tau_{c^{1 / 2}}=4.595, r_{c} \tau_{P}=4.605$, and $r_{c} \tau_{C F}=2.30,3.53,3.92$, and 4.43 for $\delta=0.1,0.5,1.0$, and 5.0, respectively (Fig. 5A). Once again, as $\delta$ increases, the $\mathrm{CF}$ model approaches the behavior of the PMA model.

\begin{tabular}{|c|c|c|c|c|}
\hline Model & $f_{P}$ & $f_{P} ; n \rightarrow 1$ & $\alpha$ & $r$ \\
\hline True mass-action (equation 6) & $\beta$ & $\beta$ & $\frac{\beta I \psi}{N}$ & $r_{W}=\beta I \psi f_{R}$ \\
\hline Pseudo mass-action (equation 7) & $\gamma n$ & $\gamma$ & $\frac{\gamma I \psi}{N_{\max }}$ & $r_{P}=\gamma I \psi f_{R}$ \\
\hline Canopy filtration (equation 17) & $n$ & 1 & $I \psi n$ & $r_{C=}=\frac{I \psi f_{R}}{}$ \\
\hline & $\overline{n+\delta}$ & $\overline{(1+\delta)}$ & $\overline{N(n+\delta)}$ & $r_{C F}=\overline{1+\delta}$ \\
\hline
\end{tabular}

The effect of time of initial infection. For most of the above epidemics, we have assumed that there was some initial level of diseased tissue at time zero. Consider the situation where the crop is allowed to grow uninfected for a certain time period, $\tau_{\text {start }}$, at which time a certain amount of disease, $Y\left(\tau_{\text {start }}\right)$, is introduced. This situation is illustrated in Figure $5 \mathrm{~B}$ for the parameters of the

TABLE 2. A comparison of the three models presented in the text $\mathrm{t}^{\mathrm{a}}$ 
CF epidemic illustrated in Figure 4A $\left(\tau_{\text {start }}=0, n(0)=0.01\right.$, $Y\left(\tau_{\text {start }}\right) / N_{\max }=0.001667, r_{C F} / r_{c}=2$, and $\left.\delta=1\right)$. For values of $\tau_{\text {start }}<$ $\tau_{C F}$, the resultant epidemics are almost identical. To illustrate this fact, note that the dimensionless time at which the diseased fraction $y$ is 0.5 are 7.10, 7.13, 7.22, 7.42, 7.83, 8.48 for values of $r_{c} \tau_{\text {start }}=0,1,2,3,4,5,6$, respectively. In this case, $r_{c} \tau_{C F}=3.92$ (equation 25) and the dimensionless time lag between $\tau_{\text {start }}=0$ and $\tau_{\text {start }}=3$ is only 0.32 . To put this information in perspective, a typical value for $r_{c}$ is $0.1 \mathrm{~d}^{-1}$, so that a 30 day delay in the introduction of inoculum results in a mere 3.2 day lag in the time for the epidemic to reach the $50 \%$ point $(y=0.5)$. Basically, disease development is delayed until the flush of crop growth.

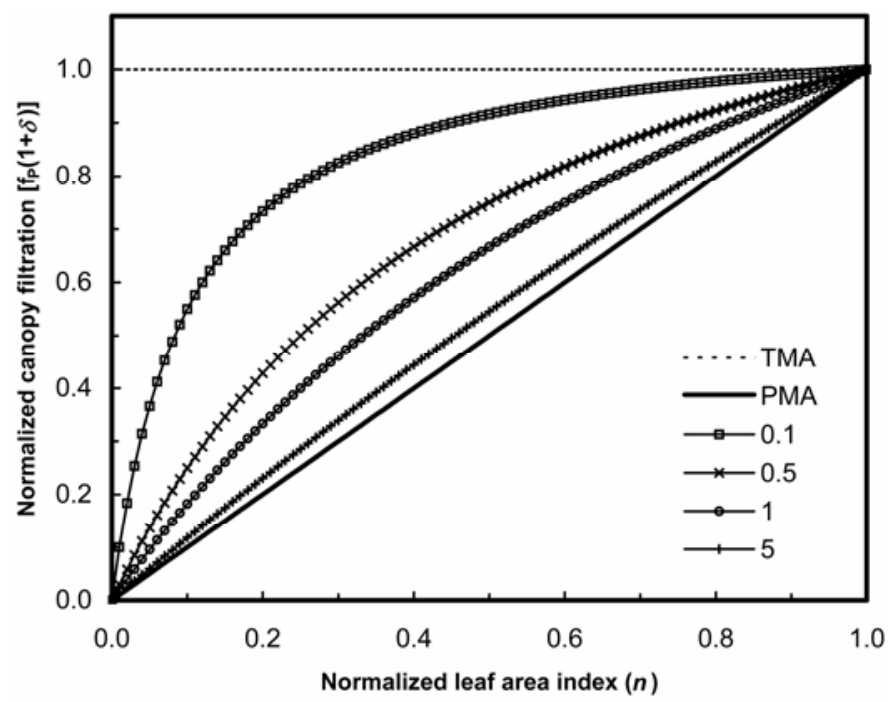

Fig. 1. The effect of crop growth on canopy filtration. The fraction of airborne spores that land on plants, $f_{P}$, divided by its maximum value $(1+\delta)^{-1}$, is plotted versus normalized leaf area index $\left(n=A / A_{\max }\right)$ for different values of the parameter $\delta$ (equation 15). The parameter $\delta$ is defined as the ratio of the number of spores lost to both the ground and the air above the canopy to the number of spores deposited on plant tissue. For comparison, the assumed canopy filtration for the true mass-action model (TMA, dashed line, equation 6) and the pseudo mass-action model (PMA, heavy solid line, equation 7) are also shown. Smaller values of $\delta$ correspond to denser canopies, faster spore settling velocities, and lower wind speeds.

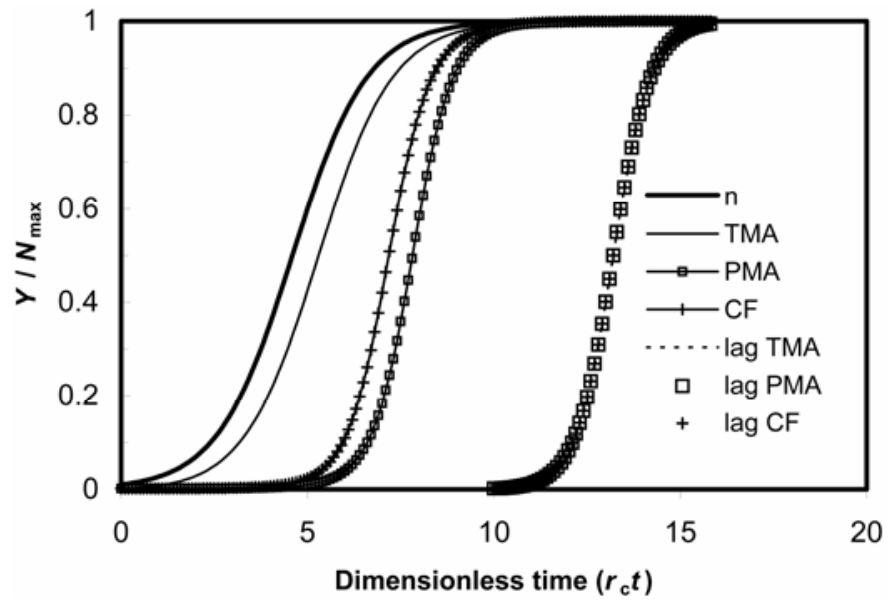

Fig. 2. Plot of the epidemics resulting from the three models discussed in the text (true mass-action [TMA, equation 6]; pseudo mass-action [PMA, equation 7]; and canopy filtration [CF, equation 17] with $r_{W}=r_{P}=r_{C F}=2 r_{c}$, $\left.Y_{0} / N_{\max }=0.00167, n_{0}=0.01, \delta=1\right) . Y$ is the cumulative number of infected sites and $N_{\max }$ is the maximum number of total sites. All three models give the same result when the epidemic is started as the crop approaches maximum ground cover $\left(r_{\mathrm{c}} t=10\right.$; lag TMA, lag PMA, lag CF). For comparison, normalized crop growth is also plotted $\left(n=N / N_{\max }\right.$; heavy line).
Population effects and lesion phenology. The above models of disease development are based on rather simplistic assumptions concerning the population of lesions and the population of host sites. First sporulating or infective sites, $S$, are assumed to be a constant fraction, $f_{R}$, of infected sites. Due to the latency period and the dependence of sporulation on the age of a lesion, the age distribution of the population of infected sites must be taken into account in order to correctly relate infective sites, $S$, to infected sites, $Y$.

Canopy filtration and the basic reproduction ratio, $R_{0}$. Segarra et al. (35: equation 4) defined the basic reproduction ratio, $R_{0}$, as

$$
R 0=\xi H_{0} \psi \int_{0}^{\infty} I(\tau) d \tau
$$

where $H_{0}$ is the total number of healthy sites (assumed susceptible) at time $0, \xi$ and $\psi$ are defined as in equation 8 , and $I(\tau)$ is the time rate of aerial spore production per lesion, as before. However, equation 27 is no longer valid for a growing crop where the total number of host sites, $N(t)$, is an explicit function of time. Crop growth (equation 5) and the canopy filtration efficiency, $f_{P}$ (equation $15)$, must be incorporated into the definition of $R_{0}$. Note the fraction of deposited spores generating lesions, $\psi\left(\right.$ lesion spore ${ }^{-1}$ ), was also assumed constant in equation 27 (35: equation 4). If $\psi$ is a function of time, it must be brought inside the time integral.

The initial product, $\xi H_{0}$, on the right side of equation 27 , by definition, is the fraction of the total number of spores which end up on the maximal number of susceptible host sites, $H_{0}$, the initial value. The analog of $\xi H_{0}$, for a growing crop, is $\xi N(t)=f_{P}[n(t), \delta]$, which is an explicit function of time. Therefore, in this case, the maximal number of available susceptible host sites is not initially present. In order to account for the increasing availability of host sites, the product, $\xi N(t)$, must be also be brought inside the time integral in equation 27. Recalling that $\xi=f_{P} / N(t)$ and assuming that all sites are healthy and susceptible, the total number of daughter lesions per mother lesion infected at time, $t_{\text {start }}$, is given by

$$
R\left(t_{\text {start }}\right)=\int_{0}^{\infty} f_{P}\left[n\left(t_{\text {start }}+\tau\right), \delta\right] \psi\left(t_{\text {start }}+\tau\right) I(\tau) d \tau
$$

where $f_{P}$ and $\delta$ are defined in equation 15 . In writing equation 28 , I have, intentionally, left out the zero subscript on " $R\left(t_{\text {start }}\right)$ " in

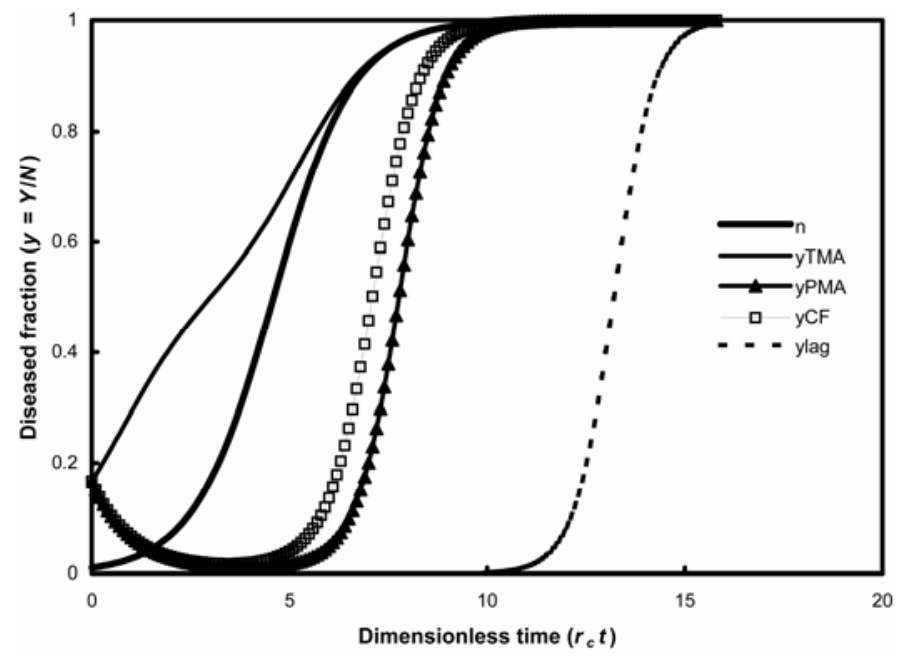

Fig. 3. Diseased fraction, $y$, versus dimensionless time, $r_{c} t$, for the epidemics plotted in Figures 2 and $3\left(r_{W}=r_{P}=r_{C F}=2 r_{c}, Y_{0} / N_{\max }=0.00167, n_{0}=0.01\right.$, $\delta=1)$. Note that $y$ decreases for the pseudo mass-action (PMA) and canopy filtration (CF) models during the early stages of the epidemic as the crop grows faster than disease increases. All three models give the same result when the epidemic is started after the crop has grown $\left(r_{c} \tau_{\text {start }}=10\right.$, ylag dashed line). For comparison, normalized crop growth is also plotted $(n=$ $N / N_{\max }$; heavy line). 
equation 28 to remind the reader that this quantity is a function of the time of lesion initiation, $t_{\text {start }}$. For most pathosystems, due to the favorable conditions for infection associated with increasing leaf area, the first two terms within the integrand of equation 28 will both, on average, be monotonically increasing functions of time throughout the vegetative phase of crop growth. There is an additional stochastic variation in $\psi$, the spore survival-infection probability, due to episodic meteorological events such as rainfall. Since a successful invasion of the pathogen requires that $R_{0}>1$
(11), there may be a threshold value for $t_{\text {start }}$ before which an epidemic is not likely to occur. In other words, lesions initiated before this critical time may not cause a self-sustainable epidemic (31).

An example: sporulation given by Vanderplank's step function. Assume that $\psi$ is constant and $I(t)$ is given by the product of a constant rate of spore release, $q$ (spores day $^{-1}$ ), and a unit step function in time, which has the value of 1 for $p>t>p+i$ and is zero at all other times (37). Thus, after a latent period of duration, $p$ (d), a new lesion produces spores at a constant rate $q$ for a
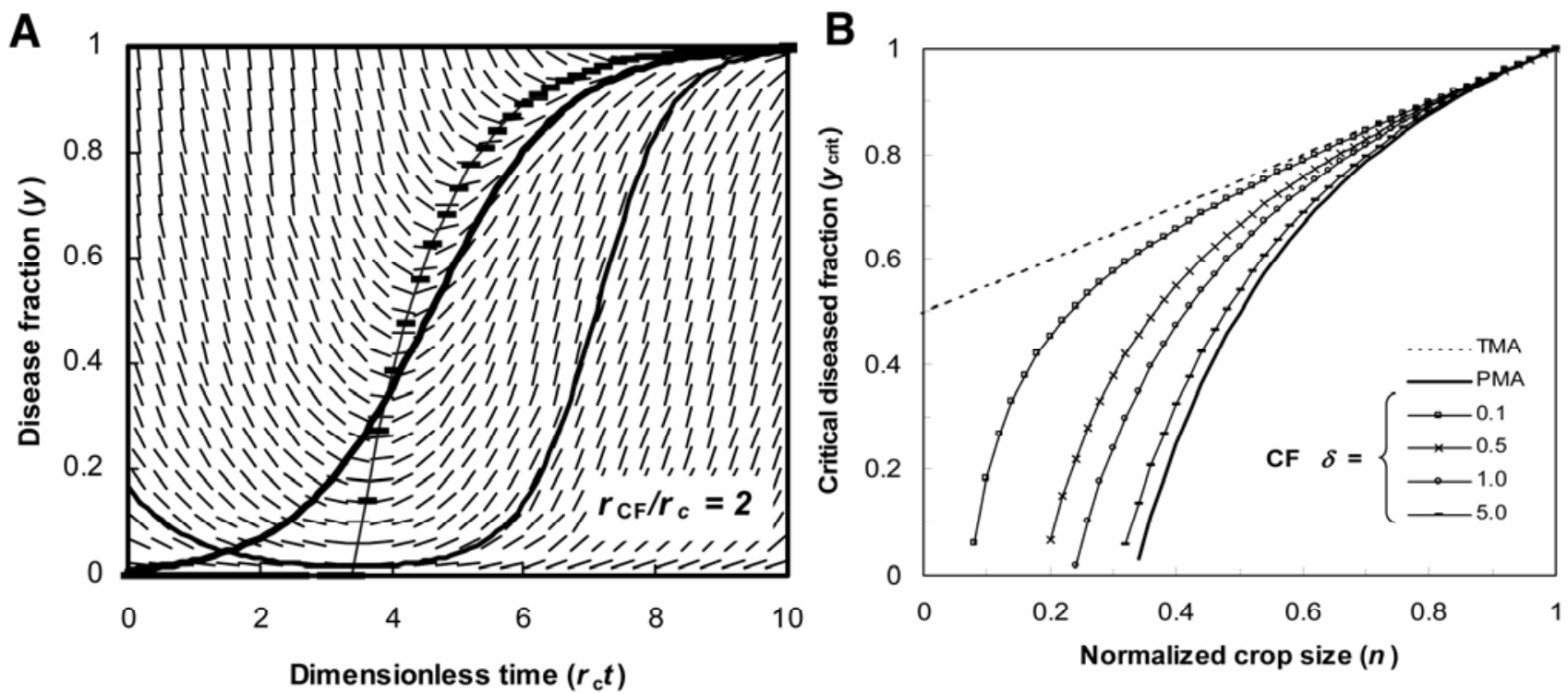

Fig. 4. A, Short line segments representing $\mathrm{d} y / \mathrm{d} t$ calculated for $r_{C F} / r_{c}=2$ and $\delta=1$ using equation 22 in text, together with equation A7 in the Appendix, are plotted versus dimensionless time, $r_{c} t$. The "U" shaped line corresponds to the $r_{C F} / r_{c}=2$ solution plotted in Figures 2 to $5 . r_{C F}\left(\mathrm{~d}^{-1}\right)$ is the maximum logistic rate of disease increase and $r_{c}\left(\mathrm{~d}^{-1}\right)$ is the logistic rate of crop growth. The hatched line is the critical diseased fraction, $y_{\text {crit }}$, given by equation 23 in the text. Note slopes are negative above and to the left of this line and positive below and to the right. The logistic curve describing the fractional leaf area, $n(t)$, is also shown for comparison (bold sigmoidal line). Figure was calculated assuming $n(0)=0.01, y(0)=0.16667$. B, The effect of crop growth on the critical diseased fraction, $y_{\text {crit }}$, calculated for the canopy filtration model (CF, equation 17), which is plotted versus normalized crop size $\left(n=N / N_{\max }=A / A_{\max }\right)$ for different values of the parameter $\delta$ (equation 23). The parameter $\delta$ is defined as the ratio of number of spores lost to both the ground and the air above the canopy to the number of spores deposited on plant tissue. For comparison, the assumed filtration for the true mass-action model (TMA, dashed line, equation 19) and the pseudo mass-action model (PMA, heavy solid line, equation 21) are also shown. Smaller values of $\delta$ correspond to denser canopies, faster falling spores, and lower wind speeds. These calculations were carried out assuming $r_{W}=r_{P}=r_{C F}=2 r_{c}$.
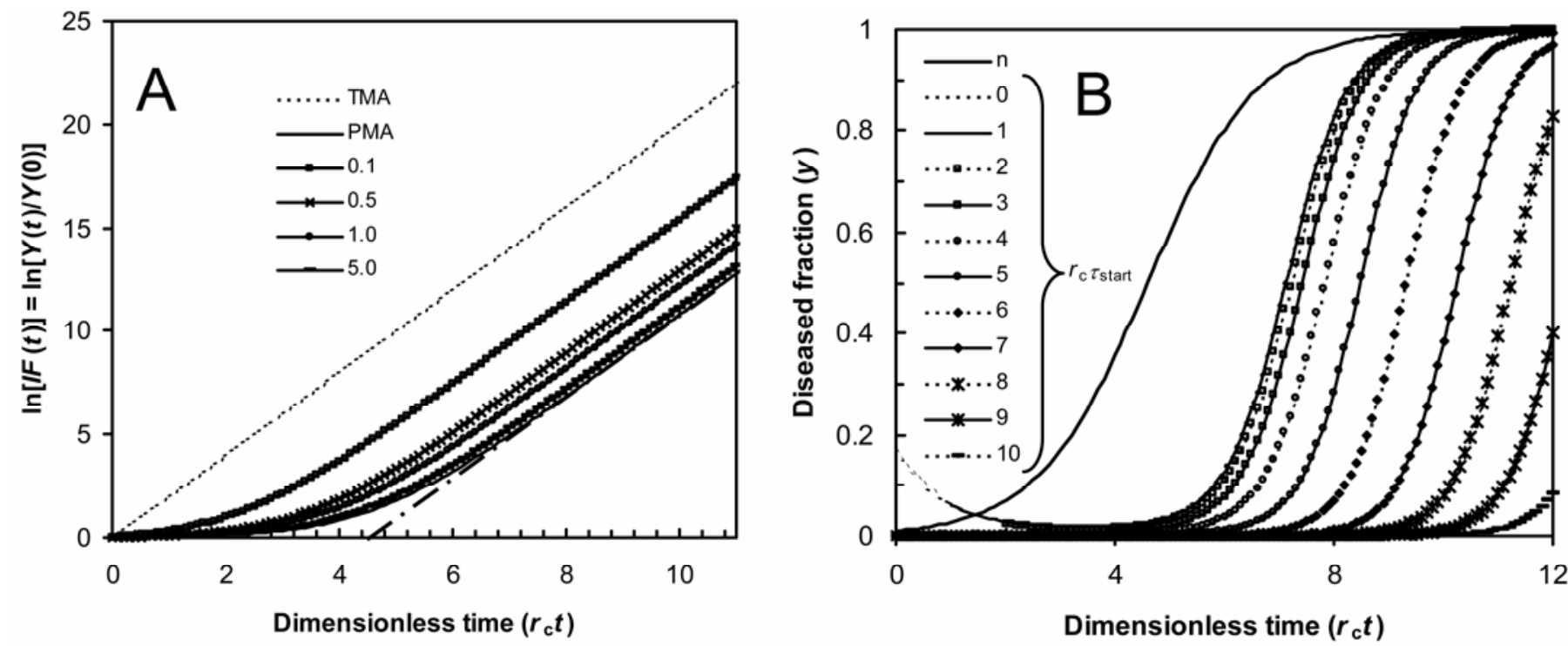

Fig. 5. A, Log plots of approximate solutions for $Y<<N$ (Appendix equations A19 to A26). A straight line indicates an exponential increase of disease. For these calculations it was assumed that $n(0)=0.01, r_{W}=r_{P}=r_{C F}=2 r_{c}$, and the asymptotic slopes of all lines is 2 . The pseudo mass-action (PMA) solution (heavy line) has the maximum dimensionless delay time, $r_{c} \tau_{P}$, equal to the natural logarithm of 100 or, approximately, 4.6 (note $r_{c} \tau_{P}=-\ln (0.01)$, equation A25). The asymptotic line for the PMA solution is also shown (dot-dash line). B, Plot of diseased fraction, $y$, versus dimensionless time, $r_{c} t$, illustrating the effect of the time of initial infection $\left(\tau_{\text {start }}\right)$ on the canopy filtration $(\mathrm{CF})$ epidemic illustrated in Figure $6\left(\tau_{\text {start }}=0, n(0)=0.01, Y\left(\tau_{\text {start }}\right) / N_{\max }=0.001667, r_{C F} / r_{c}=2\right.$, and $\left.\delta=1\right)$. The dimensionless delay time, $r_{c} \tau_{C F}$, for this epidemic is 3.92 (equation 25). As the initial inoculum is introduced later and later, very little happens until $r_{c} \tau_{\text {start }}>r_{c} \tau_{C F}$. 
period of time equal to the infectious period, $i$ (d). The total amount of spores produced over this time period is equal to the product iq. Then, using equation 15 for $f_{P}$, equation 28 can be integrated to yield

$$
R\left(t_{\text {start }}\right)=\psi q \int_{p}^{p+i} \frac{d \tau}{1+\frac{\delta}{n\left(t_{\text {start }}+\tau\right)}}=\frac{R 0}{r_{c} i} \ln \left(\frac{e^{r_{c} i}+C_{3} \exp \left[-r_{c}\left(t_{\text {start }}+p\right)\right]}{1+C_{3} \exp \left[-r_{c}\left(t_{\text {start }}+p\right)\right]}\right)
$$

where the maximum value for $f_{P}$ is $(1+\delta)^{-1}$, which when inserted into equation 27, yields $R_{0}=i \psi q /(1+\delta)$, and

$$
C_{3}=\left[\frac{\delta(1-n(0))}{(1+\delta) n(0)}\right]
$$

which was defined in equation $\mathrm{A} 16$. The ratio $R\left(t_{\mathrm{star}}\right) / R_{0}$ is plotted versus $r_{c} t_{\text {start }}$ in Figure $6 \mathrm{~A}$ and $\mathrm{B}$ for $\delta=1$. In Figure $6 \mathrm{~A}$, the value of the ratio of the infectious period to the latent period, $i / p$, is set equal to 1 and $r_{c} p$ is varied from 0.25 to 2 . In Figure 6B, the value of the ratio $r_{c} p$ is set equal to 1 and $i / p$ is varied from 0.1 to 4 . In general, an increase in either $p$ or $i$ reduces the impact of the lesion start time on the effective reproductive ratio $\left(R\left(t_{\text {start }}\right)\right)$.

The Kermack-McKendrick epidemic model. The above specification of the relations between lesion age, spore production, transport, and deposition used in the derivation of $R_{0}$ (equation 27) suggests a restatement of the mass-action equation (equation 1 ). In particular, the product $\alpha S$ can be expressed as

$$
\alpha S=\xi \psi \int_{0}^{t} \frac{d Y(t-\tau)}{d t} I(\tau) d \tau
$$

where $\tau$ is the age of a lesion, $I(\tau)$ is the time rate of spore production per lesion as a function of lesion age, $\xi$ is the probability per susceptible uninfected host site that a spore is physically transported to such a site, and $\psi$ is the fraction of spores that having landed on susceptible host cause a new lesion. The derivative in equation 31 represents the rate at which lesions were being initiated at time $t-\tau$. With all the above assumptions, equations 1 and 31 can then be combined to yield

$$
\frac{d Y(t)}{d t}=\xi H(t) \psi\left[\int_{0}^{t} \frac{d Y(t-\tau)}{d t} I(\tau) d \tau+g(t)\right]
$$

where $g(t)$ is the spore production of lesions initiated before $t=0$ and all healthy tissue is assumed susceptible. Equation 32, as written, still includes two population variables, $Y$ and $H$. Thus, an explicit solution of equation 32 involves some assumption about the relation between these two variables. Following Segarra et al. (35), we assume that the total number of host sites remains constant and is given by $N_{0}=H+Y$, such that $\mathrm{d} H / \mathrm{d} t=-\mathrm{d} Y / \mathrm{d} t$, which, when combined with equation 32 , gives the classic form of the Kermack-McKendrick epidemic model (KM model: 23):

$$
\frac{d H(t)}{d t}=\xi H(t) \psi\left[\int_{0}^{t} \frac{d H(t-\tau)}{d t} I(\tau) d \tau-g(t)\right]
$$

The solution of equation 33 results in a disease progress curve that is totally determined by the value of $R_{0}$ (equation 27), the sporulation curve $(I(\tau))$, and the time rate of spore production from the initial infection $(g(t))$.

Our task at hand is to modify the above analysis to include a growing crop for which the total number of standing sites, $N(t)$, is an explicit function of time. For equation 32, this process is simple. Expressing the parameter $\xi$ in equation 32 as an explicit function of time, $\xi=f_{P} / N(t)$, and ignoring removals such that $N=$ $H+Y$, yields

$$
\frac{d Y(t)}{d t}=f_{P} \psi\left[1-\frac{Y(t)}{N(t)}\right]\left[\int_{0}^{t} \frac{d Y(t-\tau)}{d t} I(\tau) d \tau+g(t)\right]
$$

The derivation of equation 33 involved an assumption concerning the time derivatives of $H$ and $Y$ which is no longer valid for a growing crop. Instead, ignoring removals, we have

$$
N(t)=H(t)+Y(t) \rightarrow \frac{d N(t)}{d t}=\frac{d H(t)}{d t}+\frac{d Y(t)}{d t}
$$

so that, equation 33 can be expressed as

$$
\frac{d[N(t)-H(t)]}{d t}=\frac{f_{P} \psi H(t)}{N(t)}\left[\int_{0}^{t} \frac{d[N(t-\tau)-H(t-\tau)]}{d t} I(\tau) d \tau+g(t)\right]
$$

or

$$
\frac{d H(t)}{d t}=\frac{f_{P} \psi H(t)}{N(t)}\left[\int_{0}^{t} \frac{d H(t-\tau)}{d t} I(\tau) d \tau-g(t)\right]+\frac{d N(t)}{d t}-\frac{f_{P} \psi H(t)}{N(t)} \int_{0}^{t} \frac{d N(t-\tau)}{d t} I(\tau) d \tau
$$

Direct comparison of equation 36 with equation 33 reveals two addition terms involving the time derivative of the total number of sites, $\mathrm{d} N / \mathrm{d} t$. Of course, if $N$ remains constant, these terms are zero and equation 36 reverts to equation 33 . The second positive term on the right side of equation 36 represents the effect of crop growth on the number of healthy sites, $H$, and the negative last term on the right side of equation 36 is a correction to the spore
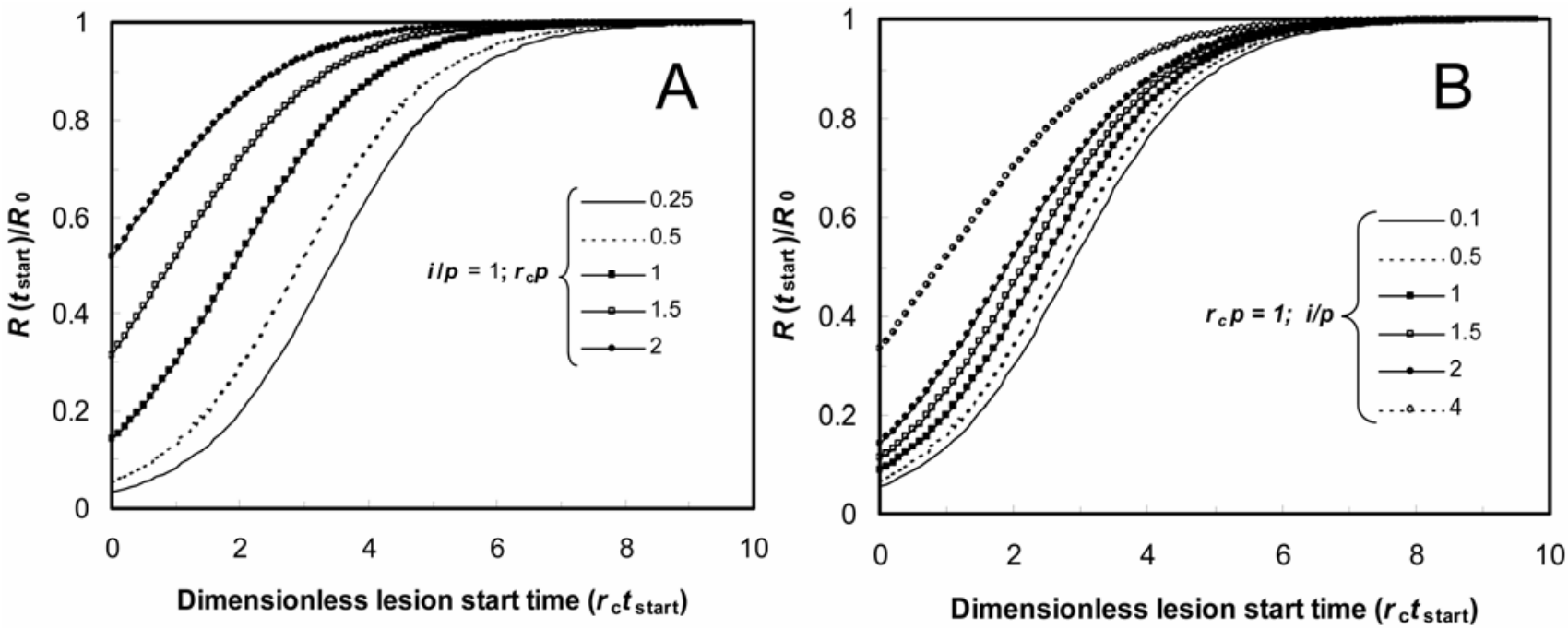

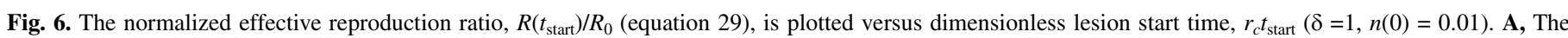

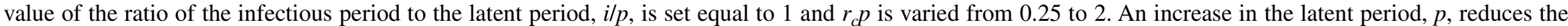

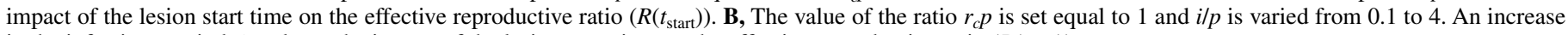
in the infectious period, $i$, reduces the impact of the lesion start time on the effective reproductive ratio $\left(R\left(t_{\text {start }}\right)\right)$. 
production term (bracketed convolution integral in the first term on the right side of equation 36) accounting for increases in $H$ due to crop growth, which is independent of $Y$ and has nothing to do with spore production.

As the above analysis indicates, the expression of epidemic development in terms of healthy sites is complicated by crop growth. In this situation, equation 34 is more useful than the modified KM model above (equation 36), since it is expressed in terms of infected sites, which are not directly affected by the introduction of a new host. For equation 34, crop growth enters explicitly in the functional dependence of $f_{P}$ on canopy density (equation 15) and the dilution effect occurring in the correction factor $(1-Y / N)$. There is no explicit dependence on $\mathrm{d} N / \mathrm{d} t$.

Lesion production days. The form of equation 28 for $R$ and the nondimensional premultiplier, $f_{P} \psi$, in equations 34 and 36 suggest the introduction of a transformed time variable, $\mathfrak{I}(t)(\mathrm{d})$, defined as

$$
\Im(t)=\int_{0}^{t} f_{P} \psi d t^{\prime}
$$

which I shall call lesion production days. If spores are produced at a constant rate of $I$ per day, and all host tissue is assumed susceptible so that there are no multiple infections, then the total number of lesions produced in time, $t$, is $I \mathfrak{I}(t)$. Dividing both sides of equation 34 by the product, $f_{P}(t) \psi(t)$, and multiplying and dividing the integrand in equation 34 by the product, $f_{P}\left(t_{\text {start }}\right) \psi\left(t_{\text {start }}\right)$, yields

$$
\frac{d Y(\mathfrak{I})}{d \mathfrak{I}}=\left[1-\frac{Y(\mathfrak{I})}{N(\mathfrak{I})}\right]\left[\int_{0}^{t} \frac{d Y\left(\mathfrak{I}_{\text {start }}\right)}{d \mathfrak{I}} I\left(\mathfrak{I}-\mathfrak{I}_{\text {start }}\right) d \mathfrak{I}+g(\mathfrak{I})\right]
$$

where $\tau=t-t_{\text {start }}$, and all time variables have been transformed using equation 37 . Thus, a simple time transformation (equation 37) can account for the time dependence in spore deposition and spore survival. There still remains the effect of dilution due to crop growth in the correction factor of equation 38 (first term on the right side), which accounts for the introduction of new healthy tissue. However, early in an epidemic, the term $Y / N$ is small and can be ignored.

If we assume that spore infection probability, $\psi$, is constant in time, then the time dependence of $\mathfrak{I}$ is identical to the time dependence of $\ln (I F)$ for the CF model (equations A15 and A24). This function, illustrated in Figure 5A, is characterized by the time lag, $\tau_{C F}$, given by equation 25 . This time lag is independent of the spore production rate and is totally given by crop-based parameters.

\section{DISCUSSION}

The epidemiological principle of pseudo mass-action (equation 7 ), wherein the number of new lesions per unit of time is proportional to the number of sporulating sites multiplied by the number of susceptible host sites, has been reexamined. For spores spread by wind, this simple linear hypothesis breaks down at high plant canopy densities. On the other hand, the simple modification of this rule, which leads to true mass-action models (equation 6 ), does not properly account for the variable efficiency with which a growing plant canopy intercepts airborne spores. A simple physical model of spore transport and deposition provided a reasonable and more realistic approximation for the changing canopy filtration efficiency. Solutions of the resulting differential equation (equations 16 and 17) for disease severity as a function of time in a growing crop provide some interesting insights.

- Disease progress is determined by two time scales, one describing crop growth and the other describing spore production rate.

- The basic reproduction ratio, $R_{0}$, defined as the number of daughter lesions per mother lesion when all host tissue is susceptible, increases dramatically during the vegetative stage of crop growth. This increase is due to increased canopy spore filtration. As the crop grows, fewer and fewer spores are deposited to the ground and/or escape from the field.

- The increase in the value of $R_{0}$, described above, can be accounted for by a transformation in the time variable.

The development of plant disease epidemics spread by airborne spores during the vegetative period is strongly dependent on canopy growth. The overall effect of the increasing canopy filtration efficiency is to delay the normal initial exponential growth of the epidemic. The magnitude of this time delay is explicitly dependent on the crop growth rate and the wind dependent canopy filtration probability. One of the ramifications of this time delay is the synchronization of early epidemics with the flush of crop growth, which is relatively independent of the time of the initial introduction of inoculum.

\section{APPENDIX}

The quadrature solution to the law of mass-action for a logistically growing crop. We seek the solution to the following set of coupled nonlinear equations:

$$
\begin{aligned}
& \frac{d Y}{d t}=\alpha f_{R} Y(N-Y) \\
& \frac{d N}{d t}=r_{c} N \frac{N_{\max }-N}{N_{\max }}
\end{aligned}
$$

The general procedure is to first solve equation 5 for $N(t)$ as an explicit function of time, which is then inserted into equation 3.

Both of the above differential equations have the same general form, that is

$$
\frac{d X}{d t}=b_{1}(t) X\left(b_{2}(t)-X\right)
$$

where $b_{1}(t)$ and $b_{2}(t)$ are arbitrary functions of time. We start by transforming equation $\mathrm{A} 1$ by setting $Z(t)=X(t)^{-1}$ such that

$$
\frac{d X}{d t}=-\frac{1}{Z^{2}} \frac{d Z}{d t}=b_{1}(t) \frac{1}{Z} \cdot\left(b_{2}(t)-\frac{1}{Z}\right)
$$

or

$$
\frac{d Z}{d t}+b_{1}(t) b_{2}(t) Z=b_{1}(t)
$$

Multiplying both sides of equation A2 by the integrating factor, $I F(t)$, defined by

$$
I F(t)=\exp \left(\int_{0}^{t} b_{1}\left(t^{\prime}\right) b_{2}\left(t^{\prime}\right) d t^{\prime}\right)
$$

renders equation A2 exact, such that

$$
\frac{d}{d t}[I F(t) Z(t)]=b_{1}(t) I F(t)
$$

yielding the following quadrature solution for equation A1:

$$
X(t)=\frac{I F(t)}{\frac{1}{X(0)}+\int_{0}^{t} b_{1}\left(t^{\prime}\right) I F\left(t^{\prime}\right) d t^{\prime}}
$$

A direct comparison of equations 5, 6, 7, and 21 with equation A1 yields

$$
\begin{array}{lll}
b_{1}=\frac{r_{c}}{N_{\max }} & ; b_{2}=N_{\max } & \text { for Eq. } 5 \\
b_{1}=\frac{r_{W}}{N(t)} & ; b_{2}=N(t) & \text { for Eq. 6 } \\
b_{1}=\frac{r_{P}}{N_{\max }} & ; b_{2}=N(t) & \text { for Eq.7 } \\
b_{1}=\frac{r_{C F}(1+\delta) f_{P}}{N(t)} & ; b_{2}=N(t) & \text { for Eq. 17 }
\end{array}
$$


Since equations 6,7, and 17 explicitly depend on $N(t)$, equation 5 must be solved first. Combining equations A3, A5, and A6 yields the following logistic equation solution for equation 5 :

$$
N(t)=N_{\max }\left(1+\frac{\left(N_{\max }-N(0)\right)}{N(0)} \exp \left(-r_{c} t\right)\right)^{-1} \text { or } n(t)=\left(1+\frac{(1-n(0))}{n(0)} \exp \left(-r_{c} t\right)\right)^{-1}
$$

where $n=N / N_{\max }$. For this case, the integrating factor (equation A3) is fairly simple $\left(\operatorname{IF}(t)=\exp \left(r_{c} t\right)\right)$.

The solution for true mass-action $\left(\alpha \propto N^{-1}\right)$. For equation 6 , we note the product $b_{1} b_{2}$ is a constant and $I F(t)=\exp \left(r_{W} t\right)$ (equation A6). So that, the integral in the denominator of equation A5 can be directly evaluated:

$$
\begin{aligned}
& \int_{0}^{t} b_{1}\left(t^{\prime}\right) I F\left(t^{\prime}\right) d t^{\prime}=\frac{r_{W}}{N_{\max }} \int_{0}^{t} \exp \left(r_{W} t^{\prime}\right) \cdot\left[1+\frac{(1-n(0))}{n(0)} \exp \left(-r_{c} t^{\prime}\right)\right] d t^{\prime} \\
& =\frac{\left[\exp \left(r_{W} t\right)-1\right]}{N_{\max }}+\frac{r_{W}}{N_{\max }\left(r_{W}-r_{c}\right)} \frac{(1-n(0))}{n(0)}\left\{\exp \left[\left(r_{W}-r_{c}\right) t\right]-1\right\} \text { for } r_{W} \neq r_{c} \\
& =\frac{\left[\exp \left(r_{W} t\right)-1\right]}{N_{\max }}+\frac{r_{W} t}{N_{\max }} \frac{(1-n(0))}{n(0)} \\
& \text { for } r_{W}=r_{c}
\end{aligned}
$$

Inserting equation A11 into equation A5, we obtain the solution to equation 6 (equation A9 in 38):

$$
\begin{array}{ll}
Y(t)=\left\{\frac{\exp \left(-r_{W} t\right)}{Y(0)}+\frac{\left[1-\exp \left(-r_{W} t\right)\right]}{N_{\max }}+\frac{r_{W}}{N_{\max }\left(r_{W}-r_{c}\right)} \frac{(1-n(0))}{n(0)}\left(e^{-r_{c} t}-e^{-r_{W} t}\right)\right\}^{-1} & \text { for } r_{W} \neq r_{c} \\
Y(t)=\left\{\frac{\exp \left(-r_{W} t\right)}{Y(0)}+\frac{\left[1-\exp \left(-r_{W} t\right)\right]}{N_{\max }}+\frac{r_{W} t \exp \left(-r_{W} t\right)}{N_{\max }} \frac{(1-n(0))}{n(0)}\right\}^{-1} & \text { for } r_{W}=r_{c}
\end{array}
$$

The solution to the law of pseudo mass-action with constant $\alpha$. In order to write the solution to equation 7 coupled with equation 5, we need to evaluate $I F(t)$ (equation A3) subject to the value of the product $b_{1} b_{2}$ (equation A6):

$$
\begin{aligned}
& \ln [I F(t)]=\int_{0}^{t} b_{1}\left(t^{\prime}\right) b_{2}\left(t^{\prime}\right) d t^{\prime}=\alpha f_{R} N_{\max } \int_{0}^{t}\left(1+\frac{(1-n(0))}{n(0)} \exp \left(-r_{c} t^{\prime}\right)\right)^{-1} d t^{\prime} \\
& \ln [I F(t)]=r_{P} \int_{0}^{t} \frac{n(0) \exp \left(r_{c} t^{\prime}\right)}{n(0) \exp \left(r_{c} t^{\prime}\right)+1-n(0)} d t^{\prime}=\frac{r_{P}}{r_{c}} \ln \left[n(0) \exp \left(r_{c} t\right)+1-n(0)\right] \\
& \therefore I F(t)=\left[n(0) \exp \left(r_{c} t\right)+1-n(0)\right]^{p / r_{c}}
\end{aligned}
$$

where $r_{P}\left(\mathrm{~d}^{-1}\right)=\alpha f_{R} N_{\max }$. Thus, the integral in the denominator of equation $\mathrm{A} 5$ can be written as

$$
\int_{0}^{t} b_{1}\left(t^{\prime}\right) I F\left(t^{\prime}\right) d t^{\prime}=\frac{r_{\boldsymbol{P}}}{N_{\max }} \int_{0}^{t}\left[n(0) \exp \left(r_{c} t^{\prime}\right)+1-n(0)\right]^{j^{p} / r_{c}} d t^{\prime}
$$

which can be integrated, in closed form, if the ratio $r_{P} / r_{c}$ is an integer, $i$ :

$$
\int_{0}^{t} b_{1}\left(t^{\prime}\right) I F\left(t^{\prime}\right) d t^{\prime}=\frac{i}{N_{\max }}\left\{(1-n(0))^{i} r_{c} t+\sum_{j=1}^{i} \frac{i !}{(i-j) \cdot j !} \frac{n(0)^{j}}{j}\left[\exp \left(j r_{c} t^{\prime}\right)-1\right] \cdot[1-n(0)]^{(i-j)}\right\}
$$

The solutions to equation 7 are obtained by inserting equations A8 and A9 into equation A5. For the special cases $r_{\mathrm{PMA}} / r_{c}=i=1$ and 2 , the solutions are shown below and the solutions for $r_{\mathrm{PMA}} / r_{c}=$ $i=1,2,3$, and 4 are plotted in Figure 1:

$$
\begin{aligned}
Y(t ; i=1)= & \frac{n(0) \exp \left(r_{c} t\right)+1-n(0)}{\frac{1}{Y(0)}+\frac{(1-n(0))}{N_{\max }} r_{c} t+\frac{n(0)\left[\exp \left(r_{c} t\right)-1\right]}{N_{\max }}} \\
Y(t ; i=2)= & \frac{\left[n(0) \exp \left(r_{c} t\right)+1-n(0)\right]^{2}}{\frac{1}{Y(0)}+\frac{2 \cdot(1-n(0))^{2}}{N_{\max }} r_{c} t+\frac{4 \cdot n(0)(1-n(0))\left[\exp \left(r_{c} t\right)-1\right]}{N_{\max }}+\frac{n(0)^{2}\left[\exp \left(2 r_{c} t\right)-1\right]}{N_{\max }}}
\end{aligned}
$$

In the general case when the ratio $r_{\mathrm{PMA}} / r_{c}$ is not an integer, the solution can be obtained by numerical integration.

The solution with variable canopy filtration. For equation 17 , the form of the integrating factor is a bit more complex. The parameter $b_{1}$ is given by

$$
b_{1}=\frac{I \psi f_{R} n}{(n+\delta) N(t)}=\frac{I \psi f_{R}}{N_{\max }}\left[\frac{n(0)+(1-n(0)) e^{-r_{c} t}}{n(0) \cdot(1+\delta)+\delta(1-n(0)) e^{-r_{c} t}}\right]
$$

and product $b_{1} b_{2}$ (equation A6) is given by

$$
b_{1} \cdot b_{2}=\frac{I \psi f_{R}}{(1+\delta / n)}=\frac{I \psi f_{R}}{\left[1+\delta \cdot\left(1+\frac{(1-n(0))}{n(0)} \exp \left(-r_{c} t\right)\right)\right]}=\frac{I \psi f_{R} n(0)}{\left[n(0)(1+\delta)+\delta(1-n(0)) e^{-r_{c}}\right]}
$$

In order to write the solution to equation 17 coupled with equation 5, we need to evaluate $I F(t)$ (equation A3) subject to the value of the product $b_{1} b_{2}$ (equation A14). The result is

$$
\begin{aligned}
\ln [I F(t)] & =I \psi f_{R} n(0) \int_{0}^{t}\left[n(0)(1+\delta)+\delta(1-n(0)) e^{-r_{c} t}\right]^{-1} d t^{\prime} \\
& =\frac{I \psi f_{R}}{r_{c}(1+\delta)} \ln \left[\frac{n(0)(1+\delta) e^{r_{c} t}+\delta(1-n(0))}{n(0)+\delta}\right]
\end{aligned}
$$

thus :

$$
I F(t)=\left[\frac{n(0)(1+\delta) e^{r_{c} t}+\delta(1-n(0))}{n(0)+\delta}\right]^{r_{C F} / r_{c}}
$$

where $r_{C F}\left(\mathrm{~d}^{-1}\right)=I \psi f_{R} /(1+\delta)$. Thus, the integral in the denominator of equation $\mathrm{A} 5$ can be written as

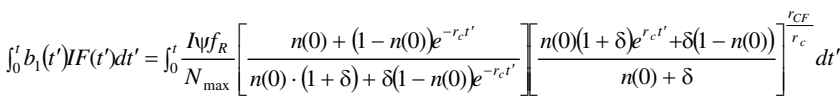

$$
\begin{aligned}
& =\frac{I \psi f_{R}}{N_{\max }(n(0)+\delta){ }^{r_{C F}} \frac{r_{c}}{r_{c}}} \int_{0}^{t}\left[n(0) e^{r_{c} t^{\prime}}+(1-n(0))\left[n(0)(1+\delta) e^{r_{c} t^{\prime}}+\delta(1-n(0))\right]^{r_{C F}} r_{c}^{r_{c}}-1 d t^{\prime}\right. \\
& =\frac{r_{C F}}{N_{\max }}\left[\frac{n(0)(1+\delta)}{n(0)+\delta}\right]^{\frac{r_{C F}}{r_{c}}} \int_{0}^{t}\left[e^{r_{c} c^{\prime}}+\frac{(1-n(0))}{n(0)}\right]\left[e^{r_{c} t^{\prime}}+\frac{\delta(1-n(0))}{(1+\delta) n(0)}\right]^{\frac{r_{C F}}{r_{c}}-1} d t^{\prime} \\
& C_{1}=\left[\frac{n(0)(1+\delta)}{n(0)+\delta}\right] \quad ; \quad C_{2}=\left[\frac{(1-n(0))}{n(0)}\right] \quad ; \quad C_{3}=\left[\frac{\delta(1-n(0))}{(1+\delta) n(0)}\right]
\end{aligned}
$$

which can be integrated in closed form when the ratio $r_{C F} / r_{c}$ is an integer $i$ :

$$
\int_{0}^{t} b_{1}\left(t^{\prime}\right) I F\left(t^{\prime}\right) d t^{\prime}=\frac{i C_{1}^{i}}{N_{\max }}\left\{C_{2} C_{3}^{i-1} r_{c} t+\sum_{j=1}^{i-1} \frac{(i-1) !}{(i-1-j) \cdot j !} C_{3}^{i-1-j}\left(\frac{C_{3}}{i-j}+\frac{C_{2}}{j}\right) \cdot\left[e^{j r_{r} t}-1\right]+\left(e^{i r_{r} t}-1\right)\right\}
$$

The solutions to equation 17 are obtained by inserting equations A15 and A17 into equation A5. For the special cases $r_{C F} / r_{c}=i=1$ and 2 , the solutions are shown below and the solutions for $r_{P} / r_{c}=$ $i=1,2,3$, and 4 are plotted in Figure 3:

$$
\begin{array}{ll}
i=1 ; & \left.Y_{1}(t)=\left\{Y(0) C_{1}\left(e^{r t}+C_{3}\right)\right]^{-1}+N_{\max }^{-1}\left(C_{2} r_{c} t+e^{r t}-1\right) \cdot\left(e^{r t}+C_{3}\right)^{-1}\right\}^{-1} \\
i=2 ; & Y_{2}(t)=\left\{\left[Y(0) C_{1}^{2}\left(e^{r t}+C_{3}\right)^{2}\right]^{-1}+2 N_{\max }^{-1}\left[C_{2} C_{3} r_{c} t+\left(C_{2}+C_{3}\right) \cdot\left(e^{r t}-1\right)+\frac{\left(e^{2 r t}-1\right)}{2}\right] \cdot\left(e^{r t}+C_{3}\right)^{-2}\right\}^{-1}
\end{array}
$$

In the general case when the ratio $r_{C F} / r_{c}$ is not an integer, the solution can be obtained by numerical integration.

Estimating the time lag. Let us reexamine equation A2:

$$
\frac{d Z}{d t}+b_{1}(t) b_{2}(t) Z=b_{1}(t)
$$

dividing by the product $b_{1} b_{2}$, we obtain

$$
\frac{d Z}{b_{1}(t) b_{2}(t) d t}=-Z+\frac{1}{b_{2}(t)}
$$


For equations 6,7 , and $17, b_{2}=N(t)$, the total number of host sites. If we assume a very low incidence of disease at the start of the epidemic, then $Y(0)<<N(0)$ and thus $Z(0)>>1 / b_{2}(t)$. Neglecting the second term on the right side of equation A19, and setting $Z=1 / Y$, we obtain

$$
\frac{d[\ln (Z)]}{d t}=-b_{1}(t) b_{2}(t)=-\frac{d[\ln (Y)]}{d t}=-\frac{d[\ln (I F)]}{d t}
$$

which implies

$$
\ln \left[\frac{Y(t)}{Y(0)}\right]=\ln [I F(t)]
$$

since $\operatorname{IF}(0)=1$ (equation A3). Equation A21 is valid as long as disease incidence is low $(Y<<N)$. For the above three models, equation $\mathrm{A} 21$ becomes

$$
\begin{aligned}
& \ln \left[\frac{Y_{W}(t)}{Y_{W}(0)}\right]=r_{W} t \\
& \ln \left[\frac{Y_{P M A}(t)}{Y_{P M A}(0)}\right]=\frac{r_{P}}{r_{c}} \ln \left[n(0) \exp \left(r_{c} t\right)+1-n(0)\right] \\
& \ln \left[\frac{Y_{C F}(t)}{Y_{C F}(0)}\right]=\frac{r_{C F}}{r_{c}} \ln \left[\frac{n(0)(1+\delta) \exp \left(r_{c} t\right)+\delta(1-n(0))}{n(0)+\delta}\right]=\frac{r_{C F}}{r_{c}} \ln \left[C_{1} \exp \left(r_{c} t\right)+1-C_{1}\right]
\end{aligned}
$$

where $C_{1}$ was defined in equations A16. Equation A22 is linear in time and equations A23 and A24 approach temporal linearity asymptotically (Fig. 5A) after time lags, $\tau_{P}(\mathrm{~d})$ and $\tau_{C F}(\mathrm{~d})$, respectively, given by

$$
\tau_{P}=-\frac{\ln [n(0)]}{r_{c}}
$$

and

$$
\tau_{C F}=-\frac{\ln \left[\frac{n(0)(1+\delta)}{n(0)+\delta}\right]}{r_{c}}=-\frac{\ln \left(C_{1}\right)}{r_{c}}
$$

\section{LITERATURE CITED}

1. Anderson, R. M., and May, R. M. 1986. The invasion, persistence, and spread of infectious diseases within animal and plant communities. Phil. Trans. R. Soc. Lond. B 314:533-570.

2. Aylor, D. E. 1998. The aerobiology of apple scab. Plant Dis. 82:838-849.

3. Aylor, D. E. 1999. Biophysical scaling and the passive dispersal of fungal spores: Relationship to integrated pest management strategies. Agric. For. Meteorol. 97:275-292.

4. Aylor, D. E., and Kyomoto, R. K. 1993. Relationship between aerial concentration of Venturia inaequalis ascospores and development of apple scab. Agric. For. Meteorol. 63:133-147.

5. Berger, R. D. 1975. Disease incidence and infection rates of Cercospora apii in plant spacing plots. Phytopathology 65:485-487.

6. Berger, R. D., and Jones, J. W. 1985. A general model for disease progress with functions for variable latency and lesion expansion on growing host plants. Phytopathology 75:792-797.

7. Burdon, J. J., and Chilvers, G. A. 1976. Controlled environment experiments on epidemics of barley mildew in different density host stands. Oecologia 26:61-72.

8. Century, K. S., Lagman, R. A., Adkisson, M., Morlan, J., Tobias, R., Schwartz, K., Smith, A., Love, J., Ronald, P. C., and Whalen, M. C. 1999. Developmental control of Xa21-mediated disease resistance in rice. Plant J. 20(2):231.

9. Chan, M. S., and Jeger, M. J. 1994. An analytical model of plant virus disease dynamics with roguing and replanting. J. Appl. Ecol. 31:413-427.

10. de Jong, M. C. M., Diekman, O., and Heesterbeek, H. 1995. How does transmission and infection depend on population size? Pages 84-94 in: Epidemic Models: Their Structure and Relation to Data. D. Mollison, ed. Cambridge University Press, Cambridge, UK.
11. Diekman, O., Heesterbeek, J. A. P., and Metz, J. A. J. 1990. On the definition and the computation of the basic reproduction ratio Ro in models for infectious diseases in heterogeneous populations. J. Math. Biol. 28:365-382.

12. Ferrandino, F. J. 1989. Spatial and temporal variation of a defoliating plant disease and reduction in yield. Agric. For. Meteorol. 47:273-290.

13. Ferrandino, F. J., and Aylor, D. E. 1985. An explicit equation for the deposition velocity. Bound. Layer Meteorol. 31:197-201.

14. Ferrandino, F. J., and Elmer, W. H.1992. Reduction in tomato yield due to Septoria leaf spot. Plant Dis. 76:208-211.

15. Ferrandino, F. J., and Elmer, W. H. 1996. Septoria leaf spot lesion density on trap plants exposed at varying distances from infected tomatoes. Plant Dis. 80:1059-1062.

16. Ficke, A., Gadoury, D. M., and Seem, R. C. 2002. Ontogenic resistance and plant disease management: A case study of grape powdery mildew. Phytopathology 92:671-675.

17. Getz, W. M., and Lloyd-Smith, W. M. 2005. Basic methods for modeling the invasion and spread of contagious diseases. DIMACS Series in Discrete Mathematics and Theoretical Computer Science. American Mathematical Society, DIMACS Center, Rutgers University, Piscataway, NJ.

18. Goudriaan, J., and Monteith, J. L. 1990. A mathematical function for crop growth based on light interception and leaf area expansion. Ann. Bot. 66:695-701.

19. Goudriaan, J., and Van Laar, H. H. 1994. Modeling Potential Crop Growth Processes. Springer Publishing Co., New York.

20. Heesterbeek, J. A. P. 2002. A brief history of R0 and a recipe for its calculation. Acta Biotheor. 50:189-204.

21. Jeger, M. J. 1982. The relation between total, infectious, and postinfectious diseased plant tissue. Phytopathology 72:1185-1189.

22. Jeger, M. J., and van den Bosch, F. 1994. Threshold criteria for model plant disease epidemics. II. Persistence and endemicity. Phytopathology 84:28-30.

23. Kermack, W. O., and McKendrick, A. G. 1927. A contribution to the mathematical theory of epidemics. Proc. R. Soc. A 115:700-721.

24. Lalancette, N., and Hickey, K. D. 1986. Disease progress as a function of plant growth. Phytopathology 76:1171-1175.

25. Lalancette, N., and Hickey, K. D. 1986. An apple powdery mildew model based on plant growth, primary inoculum, and fungicide concentration. Phytopathology 76:1176-1182.

26. Madden, L. V. 2006. Botanical epidemiology: Some key advances and its continuing role in disease management. Eur. J. Plant Pathol. 115:3-23.

27. May, R. M. 1990. Population biology and population genetics of plantpathogen associations. Pages 309-325 in: Pests, Pathogens and Plant Communities. J. J. Burdon and S. R. Leather, eds. Blackwell Scientific Publications, Oxford.

28. McCallum, H., Barlow, N., and Hone, J. 2001. How should pathogen transmission be modeled? Trends Ecol. Evol. 16(6):295-300.

29. Michaelis, L., and Menten, M. L. 1913. Kinetics of invertase action. Biochem. Z. 49:333-369.

30. Monin, A. S., and Yaglom, A. M. 1971. Statistical Fluid Mechanics: Mechanics of Turbulence. M.I.T. Press, Cambridge.

31. Onstad, D. W. 1992. Evaluation of epidemiological thresholds and asymptotes with variable plant densities. Phytopathology 82:1028-1032.

32. Onstad, D. W., and Kornkven, E. A. 1992. Persistence and endemicity of pathogens in plant populations over time and space. Phytopathology 82:561-566.

33. Raupach, M. R. 1994. Simplified expressions for vegetation roughness length and zero-plane displacement as functions of canopy height and area index. Bound. Layer Meteorol. 71:211-216.

34. Savary, S., De Jong, P. D., Rabbinge, R., and Zadoks, J. C. 1990. Dynamic simulation of groundnut rust: A preliminary model. Agric. Sys. 32:113-141.

35. Segarra, J., Jeger, M. J., and van den Bosch, F. 2001. Epidemic dynamics and patterns of plant diseases. Phytopathology 91:1001-1010.

36. Thornley, J. H. M. 1990. A new formulation of the logistic growth equation and its application to leaf area growth. Ann. Bot. 66:309-311.

37. Vanderplank, J. E. 1963. Plant Disease: Epidemics and Control. Academic Press, Inc., New York.

38. Waggoner, P. E. 1986. Progress curves of foliar diseases: Interpretation and use. Pages 3-37 in: Plant Disease Epidemiology. K. J. Leonard and W. E. Fry, eds. MacMillan Publishing Co., New York.

39. Wilson, E. B., and Worcester, J. 1945. The law of mass action in epidemiology. Proc. Natl. Acad. Sci. 31:24-34.

40. Zadoks, J. C. 1971. Systems analysis and the dynamics of epidemics. Phytopathology 61:600-610. 